\title{
Effects of Fibronectin 1 on Cell Proliferation, Senescence and Apoptosis of Human Glioma Cells Through the PI3K/AKT Signaling Pathway
}

Yu-Xiang Liao Zhi-Ping Zhang Jie Zhao Jing-Ping Liu

Department of Neurosurgery, Xiangya Hospital, Central South University, Changsha, P.R. China 